\title{
Prospective Evaluation of Changes in Tumor Size and Tumor Metabolism in Patients with Advanced Gastric Cancer Undergoing Chemotherapy: Association and Clinical Implication
}

\author{
Seongyeol Park*1, Seunggyun Ha*2, Hyun Woo Kwon ${ }^{2}$, Woo Hyoung Kim² ${ }^{2}$, Tae-Yong Kim ${ }^{1}$, Do-Youn Oh$^{1,3}$, \\ Gi Jeong Cheon ${ }^{2,3}$, and Yung-Jue Bang ${ }^{1,3}$ \\ ${ }^{I}$ Department of Internal Medicine, Seoul National University Hospital, Seoul National University College of Medicine, Seoul, Korea; \\ ${ }^{2}$ Department of Nuclear Medicine, Seoul National University Hospital, Seoul National University College of Medicine, Seoul, Korea; \\ and ${ }^{3}$ Cancer Research Institute, Seoul National University College of Medicine, Seoul, Korea
}

A change in tumor size is a well-validated and commonly used value for evaluating response to chemotherapy in cancer. Metabolic changes induced by chemotherapy are related to prognosis in several tumor types. However, the clinical implication of metabolic changes in patients with advanced gastric cancer (AGC) undergoing chemotherapy remains unclear. We aimed to evaluate response of tumor size and metabolism in AGC during chemotherapy and to reveal the relationship between them in view of their impact on patient survival. Methods: We prospectively enrolled patients with AGC before the initiation of first-line palliative chemotherapy. Using baseline and follow-up contrast-enhanced CT and ${ }^{18}$ F-FDG PET,

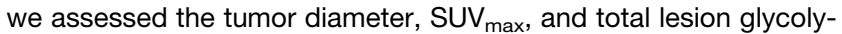
sis in each lesion and their changes during chemotherapy at the same time. We included all lesions with the maximal longest diameters over $1 \mathrm{~cm}$ on $\mathrm{CT}$, and each lesion was evaluated by matched ${ }^{18} \mathrm{~F}$-FDG PET. We analyzed the association between changes in tumor metabolism and tumor size and performed outcome analysis on overall survival (OS) and progression-free survival (PFS). Results: Seventy-four patients were enrolled, and the number of all lesions included in this study was 620 . Compared with adenocarcinomas, poorly cohesive carcinomas demonstrated lower $\mathrm{SUV}_{\max }$ irrespective of tumor size $(P<0.001)$. Human epidermal growth factor receptor 2 (HER2)-positive tumors showed higher SUV ${ }_{\max }$ than HER2-negative tumors $(P=0.002)$. The changes in SUV $\max$ due to chemotherapy had a linear correlation with the changes in tumor size of each lesion, and a $30 \%$ tumor size reduction was associated with a $50 \%$ SUV $_{\max }$ reduction $(P<0.001)$. Total lesion glycolysis changes also correlated with tumor size changes $(P<0.001)$. Better OS and PFS were obtained in patients with both tumor size and SUV $_{\max }$ reduction than in patients with either size or SUV $V_{\max }$ reduction only (OS, $P=0.003$; PFS, $P=0.038$ ). Conclusion: Changes in tumor metabolism induced by chemotherapy correlated with changes in tumor size in AGC. Considering both changes in metabolism and size could help predict a more accurate prognosis for AGC patients undergoing chemotherapy.

Received Aug. 16, 2016; revision accepted Oct. 19, 2016.

For correspondence or reprints contact either of the following:

Do-Youn Oh, Department of Internal Medicine, Seoul National

University Hospital, 101 Daehak-ro, Jongno-gu, Seoul, Korea 03080.

E-mail: ohdoyoun@snu.ac.kr

Gi Jeong Cheon, Department of Nuclear Medicine, Seoul National

University Hospital, 101 Daehak-ro, Jongno-gu, Seoul, Korea 03080.

E-mail: larrycheon@snu.ac.kr

${ }^{*}$ Contributed equally to this work.

Published online Nov. 10, 2016.

COPYRIGHT (C 2017 by the Society of Nuclear Medicine and Molecular Imaging.
Key Words: stomach neoplasm; metabolism; chemotherapy; positron-emission tomography; prognosis

J Nucl Med 2017; 58:899-904

DOI: 10.2967/jnumed.116.182675

$\mathbf{T}$ umor metabolism can be measured using ${ }^{18} \mathrm{~F}-\mathrm{FDG}$ PET, and these measurements are of significant importance in predicting the prognosis in several types of cancer (1-3). AGC is a leading cause of death worldwide, and chemotherapy and targeted therapy are the cornerstones of its treatment (4-6). Appropriate evaluation of treatment response is crucial during chemotherapy, and the current evaluation in AGC is dependent on the measurement of tumor size by $\mathrm{CT}$ according to the RECIST (7).

Several studies have shown the benefit of ${ }^{18} \mathrm{~F}-\mathrm{FDG}$ PET in the preoperative staging setting of gastric cancer, which can detect more metastatic lesions than CT (8-10). Early metabolic response of tumors determined using ${ }^{18} \mathrm{~F}-\mathrm{FDG}$ PET is correlated with histologic response and reflects the prognosis in a neoadjuvant chemotherapy setting (11-13). ${ }^{18}$ F-FDG PET can also be used to detect recurrence after surgery for gastric cancer (14).

Despite the broad diagnostic applicability of ${ }^{18} \mathrm{~F}-\mathrm{FDG}$ PET in gastric cancer, few studies have used it for response evaluation to chemotherapy in AGC. This could be attributed to the presence of well-established response evaluation methods for chemotherapy with tumor size change and to the alleged lack of a relationship between tumor metabolic response and tumor size response in AGC (7).

Therefore, the purpose of this prospective study was to evaluate response of tumor size and metabolism in AGC at the same time during chemotherapy and to reveal the exact relationship between them in view of their impact on patient survival.

\section{MATERIALS AND METHODS}

\section{Patient Population}

Patients diagnosed with AGC and candidates for first-line palliative chemotherapy were registered in this prospective cohort study at Seoul National University Hospital. Patients underwent ${ }^{18}$ F-FDG PET and corresponding contrast-enhanced CT scanning before the initiation of chemotherapy and at the time of first tumor response evaluation of the chemotherapy. The response evaluation was done after $6 \mathrm{wk}$ of chemotherapy - that is, after 2 cycles of XELOX (capecitabine and oxaliplatin Q 3 wk) chemotherapy or 3 cycles of FOLFOX (fluorouracil 
with leucovorin and oxaliplatin Q 2 wk) chemotherapy. Clinicopathologic characteristics and survival data were also collected.

The study was approved by the Institutional Review Board of Seoul National University Hospital (H-1307-132-508), and all subjects signed an informed consent form. All procedures used in this study were performed in accordance with the guidelines of the Helsinki Declaration and its amendments.

\section{PET/CT Protocol}

Dedicated PET/CT scanners (Biograph True-Point, Biograph mCT 40, and Biograph mCT 64; Siemens) were used to acquire ${ }^{18} \mathrm{~F}$-FDG PET/CT images. All patients fasted at least $6 \mathrm{~h}$ and maintained blood sugar levels less than $210 \mathrm{mg} / \mathrm{dL}$ before the injection of $5.18 \mathrm{MBq} / \mathrm{kg}$ of ${ }^{18} \mathrm{~F}-\mathrm{FDG}$. PET/CT images were acquired $1 \mathrm{~h}$ (mean, $65 \mathrm{~min}$; range, 54-87 min; SD, 8) after ${ }^{18}$ F-FDG injection. The ordered-subsets expectation maximization algorithm (4 iterations and 8 subsets for Biograph True Point; 2 iterations and 21 subsets for Biograph mCTs) was applied to reconstruct the acquired images. A 5.0-mm gaussian filter was used to postprocess the reconstructed images to reduce noise and smoothen image quality. Images were analyzed using a commercial software program (Syngo.via; Siemens Medical Solutions).

\section{Measurement of Lesions}

All measurable lesions with the short-axis diameters of lymph nodes and long-axis diameters of all other lesions greater than $1 \mathrm{~cm}$ on CT were included, regardless of target lesion definition according to the RECIST. Lesions with dimensions smaller than $1 \mathrm{~cm}$ and lesions that could not be measured reproducibly on $\mathrm{CT}$ due to their irregular conformation were excluded (7). Therefore, primary gastric lesion without protruding mass, peritoneal involvement without sizable nodule, lymphangitic pulmonary metastasis, and malignant pleural effusion, which were observed in 55 (74.3\%), 14 (18.9\%), 3 (4.1\%), and 3 (4.1\%) patients, respectively, were not included in the analyses.

The maximal longest diameter of each lesion was measured on the axial plane of the CT, which has a slice thickness of $5 \mathrm{~mm}$ or less. For each lesion measured on CT, a volume of interest was placed to cover the entire tumor sufficiently on the PET scan. Tumors were segmented using a fixed threshold SUV of 2.5 in the volume of interest. The metabolic tumor volume was defined as voxel volume multiplied by voxel number in the segmented tumor on the PET scan. The average of SUVs of voxels in segmented tumor was defined as $\mathrm{SUV}_{\text {mean }}$. The $\mathrm{SUV}_{\max }$ and total lesion glycolysis at an SUV of 2.5 (TLG2.5), calculated by multiplying the $\mathrm{SUV}_{\text {mean }}$ and metabolic tumor volume using threshold SUVs of 2.5, were measured using PET. Measurements were repeated during follow-up imaging at the time of initial response evaluation. The mean values of size, $\mathrm{SUV}_{\max }$, and TLG2.5 of all lesions included in this study were compared between baseline and initial follow-up imaging of first-line palliative chemotherapy.

\section{Statistical Analysis}

Patients were divided into several groups according to clinicopathologic factors, and mean tumor size and metabolism in each group were compared using the Student $t$ test. The relationship between the mean change in tumor size and $\mathrm{SUV}_{\text {max }}$ or TLG2.5 was analyzed using Pearson correlation analyses and scatterplots, and linear regression analyses were performed to obtain linear regression equations and $R^{2}$ values. Cutoff values of $\mathrm{SUV}_{\max }$ or TLG2.5 that predicted a tumor size reduction of $30 \%$ were found using these linear regression equations. OS and PFS were compared using Kaplan-Meier curves and log-rank tests among the groups. OS was defined as the time from initiating treatment to death, and PFS was defined as the time from initiating treatment to disease progression or any cause of death. Analyses were conducted using SPSS Statistics for Windows (version 20.0; IBM Corp.).

\section{RESULTS}

\section{Characteristics of Patients and Tumors}

Seventy-four patients were included in this study (Table 1). The median age of the patients was 61 y (age range, 28-82 y), and 53 patients $(71.6 \%)$ were men. According to histologic types, most tumors were adenocarcinomas $(79.7 \%) ; 20.3 \%$ of patients had poorly cohesive carcinomas. Fifty-six percent of adenocarcinomas were well to moderately differentiated, and $44 \%$ were poorly differentiated. Human epidermal growth factor receptor 2 (HER2) positivity was observed in $18.9 \%$ of the patients. Regimens for first-line chemotherapy comprised fluorouracil/leucovorin/oxaliplatin (41.9\%), capecitabine/

TABLE 1

Patient Characteristics $(n=74)$

\begin{tabular}{ll}
\hline \multicolumn{1}{c}{ Characteristic } & \multicolumn{1}{c}{ Value } \\
\hline Median age $(y)$ & 61 (range, 28-82) \\
Sex & $53(71.6)$ \\
\hline Male & $21(28.4)$ \\
\hline Female & \\
\hline Histologic type & $59(79.7)$ \\
\hline Adenocarcinoma & $15(20.3)$ \\
\hline Poorly cohesive carcinoma & \\
\hline Differentiation ( $n=59)$ & $33(55.9)$ \\
\hline Well to moderate & $26(44.1)$ \\
\hline Poor & $60(81.1)$ \\
HER2 status & $14(18.9)$ \\
\hline
\end{tabular}

Having primary lesion of the stomach

\begin{tabular}{|c|c|}
\hline No & $15(20.3)$ \\
\hline Yes & $59 *(79.7)$ \\
\hline \multicolumn{2}{|l|}{ ECOG PS } \\
\hline 0,1 & $65(87.8)$ \\
\hline 2,3 & $9(12.2)$ \\
\hline \multicolumn{2}{|l|}{ Chemotherapy regimen } \\
\hline FOLFOX & 31 (41.9) \\
\hline XELOX & $23(31.1)$ \\
\hline $\mathrm{XP}+\mathrm{H}$ & $14(18.9)$ \\
\hline Other & $6(8.1)$ \\
\hline Median PFS (mo) & 8.0 (95\% Cl, 6.61-9.33) \\
\hline Response rate (\%) & 41 \\
\hline Disease control rate (\%) & 70 \\
\hline Median overall survival (mo) & $17.4(95 \% \mathrm{Cl}, 12.15-22.65)$ \\
\hline
\end{tabular}

*Number of undetected lesions in baseline PET was 3, and of reproducibly measurable lesions on CT scan it was 4 .

ECOG PS = Eastern Cooperative Oncology Group Performance Status; FOLFOX = fluorouracil with leucovorin and oxaliplatin; XELOX = capecitabine and oxaliplatin; $\mathrm{XP}+\mathrm{H}=$ capecitabine and cisplatin and trastuzumab.

Data in parentheses are percentages, unless otherwise indicated. 
oxaliplatin $(31.1 \%)$, capecitabine/cisplatin/trastuzumab (18.9\%), and other regimens included capecitabine monotherapy or fluorouracil/leucovorin/irinotecan (8.1\%). The median number of evaluable lesions by our definition on CT was 4 , ranging from 1 to 47 per patient. A total of $59(79.7 \%)$ patients had primary gastric lesions; 55 patients were diagnosed as stage 4 with metastatic disease, therefore no surgery was performed, and 4 patients had a secondary primary gastric cancer at the remnant stomach diagnosed long after the first curative subtotal gastric resection of their initial gastric cancer. Among these 59 patients with gastric lesions, only 4 cases of gastric lesions were reproducibly measurable on CT. The median follow-up duration was 11.7 mo (range, 1.9-31.4 mo), and $37(50.0 \%)$ patients had died at the time of data cutoff. The median OS (mOS) was 17.4 mo (95\% confidence interval [CI], 12.2-22.7 mo), and the median PFS (mPFS) was 8.0 mo (95\% CI, 6.6-9.3 mo).

The total number of evaluable lesions at baseline for all patients was 620 (Supplemental Table 1; supplemental materials are available at http://jnm.snmjournals.org). Of these, 516 (83.2\%) were adenocarcinomas and the remaining $(16.8 \%)$ were poorly cohesive carcinomas. HER2-positive lesions accounted for $26.5 \%$ of all measurable lesions. There were 4 primary lesions $(0.6 \%)$, and the remaining were metastatic lesions, including 324 (52.3\%) lymph nodes, $262(42.3 \%)$ liver lesions, $11(1.8 \%)$ bone lesions, $9(1.5 \%)$ peritoneal seeding nodules, $5(0.8 \%)$ adrenal gland lesions, $4(0.6 \%)$ lesions in the adnexa of the uterus, and $1(0.2 \%)$ soft-tissue lesion.

\section{Baseline Tumor Size and Metabolism}

There was no difference in tumor size between adenocarcinomas and poorly cohesive carcinomas (mean size, 20.6 vs. $19.4 \mathrm{~mm} ; P=$ 0.468), but adenocarcinomas showed higher $\mathrm{SUV}_{\max }$ and TLG2.5 than poorly cohesive carcinomas (mean $\mathrm{SUV}_{\max }, 11.0$ vs. 7.3, $P<$ 0.001 ; mean TLG2.5, 136.7 vs. $60.8, P=0.001$ ) (Table 2). Among the adenocarcinomas, well-to-moderately differentiated tumors exhibited larger size than did poorly differentiated tumors (mean size, 22.4 vs. $18.2 \mathrm{~mm} ; P=0.001$ ). Although there was no significant difference in $\mathrm{SUV}_{\max }$ between these 2 tumor types (mean $\mathrm{SUV}_{\max }, 11.3$ vs. $\left.10.6, P=0.180\right)$, TLG2.5 was higher in well- to-moderately differentiated tumors than in poorly differentiated ones - a finding that could be explained by the difference in tumor size (mean TLG2.5, 168.5 vs. $92.1, P=0.018$ ). There was no significant difference in tumor size, $\mathrm{SUV}_{\max }$, and TLG2.5 between HER2-negative tumors and HER2-positive tumors. (mean size, 20.1 vs. $21.4 \mathrm{~mm}, P=0.398$; mean $\mathrm{SUV}_{\max }, 10.2$ vs. $11.0, P=0.172$; mean TLG2.5, 111.9 vs. $157.5, P=0.220$ ). However, compared with HER2-negative tumors, HER2-positive tumors showed a higher $\mathrm{SUV}_{\max }$ in subgroup analysis, with tumor sizes of $20 \mathrm{~mm}$ or larger (mean $\mathrm{SUV}_{\max }, 17.0$ vs. 11.9, $P=0.002$ ).

In general, lesions with a larger size had a higher $\mathrm{SUV}_{\max }$ and TLG2.5 in baseline images $\left(\mathrm{SUV}_{\max }\right.$, Pearson $r=0.33$, $P<0.001$; TLG2.5, Pearson $r=0.82, P<0.001)$. According to histologic types, adenocarcinomas showed a correlation between baseline size and $\mathrm{SUV}_{\max }$, but poorly cohesive carcinomas did not show such a correlation (adenocarcinomas, Pearson $r=0.36, P<0.001$; poorly cohesive carcinomas, Pearson $r=$ $0.14, P=0.145)$.

\section{Response of Tumor Size and Tumor Metabolism During Chemotherapy}

There was no difference in amount of changes in tumor size, $\mathrm{SUV}_{\max }$, and TLG2.5 between adenocarcinomas and poorly cohesive carcinomas after chemotherapy (Table 3). There was also no difference in response between well-to-moderately differentiated adenocarcinomas and poorly differentiated adenocarcinomas. HER2-positive tumors manifested a more dramatic response of tumor size, $\mathrm{SUV}_{\max }$, and TLG2.5 than did HER2-negative tumors (mean size change, $-50.2 \%$ vs. $-20.8 \%, P<0.001$; mean $\mathrm{SUV}_{\max }$ change, $-66.0 \%$ vs. $-45.5 \%, P<0.001$; mean TLG2.5 change, $-91.9 \%$ vs. $10.0 \%, P<0.001)$.

The change in tumor size in each lesion showed a positive correlation with a change in $\mathrm{SUV}_{\max }$ or TLG2.5. Moreover, the change in $\mathrm{SUV}_{\max }$ in each lesion showed a definite linearity with the change in tumor size in the scatterplot $\left(R^{2}=0.47, P<0.001\right)$ (Fig. 1A). Using the linear regression equation, we found that a $30 \%$ tumor size reduction was related to a $-50.3 \%(95 \% \mathrm{CI}$, $-49.4 \%$ to $-51.2 \%) \mathrm{SUV}_{\max }$ change. The change in size and

TABLE 2

Baseline Tumor Size, SUV $\max$, and TLG2.5 of Measurable Lesions According to Pathologic Features $(n=620)$

\begin{tabular}{|c|c|c|c|c|c|c|c|}
\hline Characteristic & No. & Mean tumor size $(\mathrm{mm})$ & $P^{\star}$ & Mean SUV $_{\max }$ & $P^{*}$ & Mean TLG2.5 & $P^{*}$ \\
\hline \multicolumn{8}{|l|}{ Histologic type } \\
\hline Adenocarcinoma & $516(83.2)$ & 20.6 & 0.468 & 11.0 & $<0.001$ & 136.7 & 0.001 \\
\hline Poorly cohesive carcinoma & $104(16.8)$ & 19.4 & & 7.3 & & 60.8 & \\
\hline \multicolumn{8}{|l|}{ Differentiation $(n=516)$} \\
\hline Well to moderate & $301(58.3)$ & 22.4 & 0.001 & 11.3 & 0.180 & 168.5 & 0.018 \\
\hline Poor & $215(41.7)$ & 18.2 & & 10.6 & & 92.1 & \\
\hline \multicolumn{8}{|l|}{ HER2 status for whole lesions } \\
\hline Negative & 456 (73.5) & 20.1 & 0.398 & 10.2 & 0.172 & 111.9 & 0.220 \\
\hline Positive & $164(26.5)$ & 21.4 & & 11.0 & & 157.5 & \\
\hline \multicolumn{8}{|c|}{ HER2 status for lesions $\geq 20 \mathrm{~mm}(n=179)$} \\
\hline Negative & $134(74.9)$ & 34.6 & 0.117 & 11.9 & 0.002 & 308.6 & 0.099 \\
\hline Positive & $45(25.1)$ & 41.0 & & 17.0 & & 505.9 & \\
\hline
\end{tabular}

*Student $t$ test was used to examine difference in values within each group.

Data in parentheses are percentages. 
TABLE 3

Response of Tumor Size and Tumor Metabolism in Each Lesion According to Pathologic Features $(n=620)$

\begin{tabular}{|c|c|c|c|c|c|c|c|}
\hline Characteristic & No. & $\begin{array}{c}\text { Mean tumor size } \\
\text { change }(\%)\end{array}$ & $P^{\star}$ & $\begin{array}{c}\text { Mean SUV }{ }_{\text {max }} \\
\text { change }(\%)\end{array}$ & $P^{\star}$ & $\begin{array}{c}\text { Mean TLG2.5 } \\
\text { change }(\%)\end{array}$ & $P^{\star}$ \\
\hline \multicolumn{8}{|l|}{ Histologic type } \\
\hline Adenocarcinoma & $516(83.2)$ & -28.8 & 0.750 & -51.8 & 0.301 & -8.5 & 0.299 \\
\hline Poorly cohesive carcinoma & $104(16.8)$ & -27.4 & & -46.2 & & -67.7 & \\
\hline \multicolumn{8}{|l|}{ Differentiation $(n=516)$} \\
\hline Well to moderate & $301(58.3)$ & -30.8 & 0.208 & -54.5 & 0.178 & 3.1 & 0.567 \\
\hline Poor & $215(41.7)$ & -26.1 & & -48.1 & & -24.8 & \\
\hline \multicolumn{8}{|l|}{ HER2 status } \\
\hline Negative & $456(73.5)$ & -20.8 & $<0.001$ & -45.5 & $<0.001$ & 10.0 & $<0.001$ \\
\hline Positive & $164(26.5)$ & -50.2 & & -66.0 & & -91.9 & \\
\hline
\end{tabular}

*Student $t$ test was used to examine difference in values within each group.

Data in parentheses are percentages.

TLG2.5 also showed a linear correlation, and a $30 \%$ of tumor size reduction was correlated with a $-62.3 \%(95 \% \mathrm{CI},-60.9 \%$ to $-63.6 \%)$ TLG2.5 change $\left(R^{2}=0.42, P<0.001\right)$ (Fig. 1B).

\section{Survival Analysis}

The patients were divided on the basis of the criteria of $30 \%$ tumor size reduction, 50\% $\mathrm{SUV}_{\max }$ reduction, and 60\% TLG2.5 reduction, respectively, which were equivalent values in linear regression equations. All of these reductions were significant for better OS, and size and $\mathrm{SUV}_{\max }$ reduction were significant for better PFS at individual analysis using the log-rank test (30\% size reduction: $\mathrm{mOS}, 19.2$ vs. $11.7, P=0.013$ and $\mathrm{mPFS}, 9.5$ vs. 4.9 , $P=0.028 ; 50 \% \mathrm{SUV}_{\max }$ reduction: $\mathrm{mOS}, 20.0$ vs. $10.6, P=0.003$ and mPFS, 9.3 vs. $5.8, P=0.023$; $60 \%$ TLG2.5 reduction: $\mathrm{mOS}$, 19.2 vs. $9.7, P=0.042$ and $\mathrm{mPFS}, 9.2$ vs. $4.9, P=0.216)$.

Survival analysis using both $30 \%$ tumor size reduction and $50 \%$ $\mathrm{SUV}_{\max }$ reduction was performed to examine the additive effect of $\mathrm{SUV}_{\max }$ decline in addition to size. The number of patients who achieved both $30 \%$ size and $50 \% \mathrm{SUV}_{\max }$ reduction, either size or $\mathrm{SUV}_{\max }$ reduction, and neither size nor $\mathrm{SUV}_{\max }$ reduction was 36
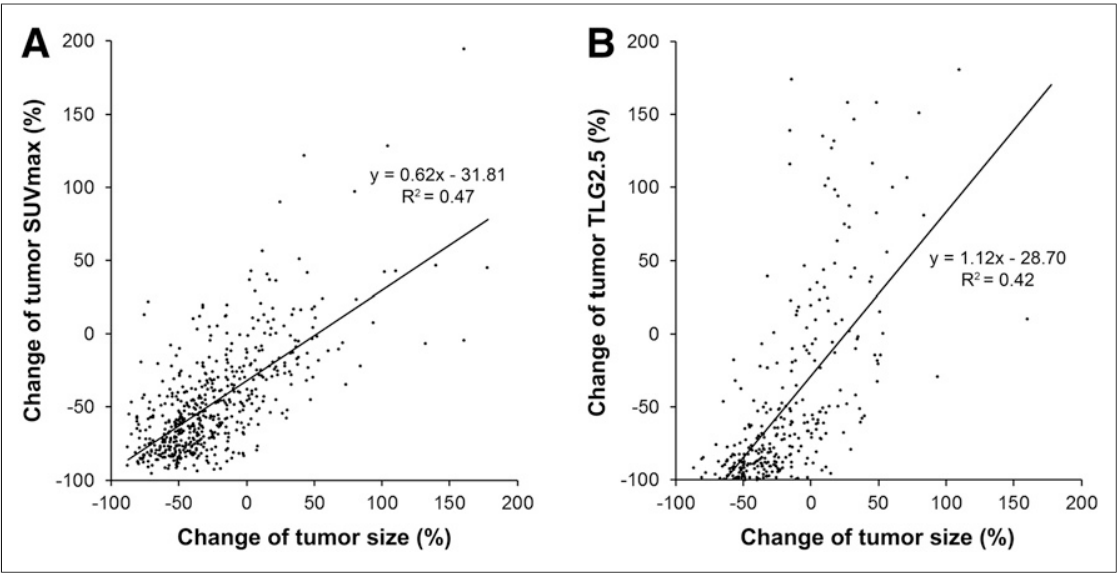

FIGURE 1. Scatterplots between change in size and SUV $\max (A)$ or TLG2.5 (B) of each individual lesion. Undetectable lesions on follow-up images $(-100 \%)$ and extreme outliers $(>200 \%)$ are omitted from linear regression analysis.
(48.6\%), 15 (20.3\%), and 23 (31.1\%), respectively. There was a significant difference in OS and PFS between the group with both size and $\mathrm{SUV}_{\max }$ reduction and the group with either size or $\mathrm{SUV}_{\max }$ reduction $(\mathrm{mOS}$, not reached vs. $11.1 \mathrm{mo}, P=0.003$; mPFS, 9.8 vs. 6.1 mo, $P=0.038$ ) (Figs. $2 \mathrm{~A}$ and $2 \mathrm{~B}$ ). However, there was no significant difference between the group with either size or $\mathrm{SUV}_{\max }$ reduction and the group with neither size nor $\mathrm{SUV}_{\max }$ reduction (mOS, 11.1 vs. 10.6 mo, $P=0.994$; mPFS, 6.1 vs. $4.9 \mathrm{mo}, P=0.562$ ).

Similarly, we performed survival analysis using the criteria of $30 \%$ tumor size reduction and $60 \%$ TLG2.5 reduction. The number of patients who achieved both size and TLG2.5 reduction, either size or TLG2.5 reduction, and neither size nor TLG2.5 reduction was $43(60.6 \%), 8(11.3 \%)$, and $20(28.2 \%)$, respectively (initial mean TLG2.5 of 3 patients [1 patient with adenocarcinoma, and 2 patients with poorly cohesive carcinomas] was not calculable because the $\mathrm{SUV}_{\max }$ values were lower than 2.5). The results of TLG2.5 showed a tendency similar to those of $\mathrm{SUV}_{\max }$, but the statistical power of TLG2.5 was lower than that of $\mathrm{SUV}_{\max }$ because of the small number of patients in the group with either size or TLG2.5 reduction (mOS: 19.2, 10.6, and $9.8 \mathrm{mo}$; mPFS: $9.5,6.0$, and 4.9 mo in groups with both size and TLG2.5 reduction, either size or TLG2.5 reduction, and neither size nor TLG2.5 reduction, respectively). Nevertheless, there was a significant difference in OS between the groups with both size and TLG2.5 reduction and with either size or TLG2.5 reduction ( $P=0.021$ ) (Figs. $2 \mathrm{C}$ and 2D). Additional analyses with only the target lesions, which were defined according to the RECIST (version 1.1) (7), showed an even more prominent effect of size and $\mathrm{SUV}_{\max } / \mathrm{TLG} 2.5$ reduction than of size or $\mathrm{SUV}_{\max } / \mathrm{TLG} 2.5$ reduction on OS and PFS (Supplemental Fig. 1).

In multivariate Cox proportional analyses with the variables age, histologic type, HER2 status, and previous gastrectomy, 


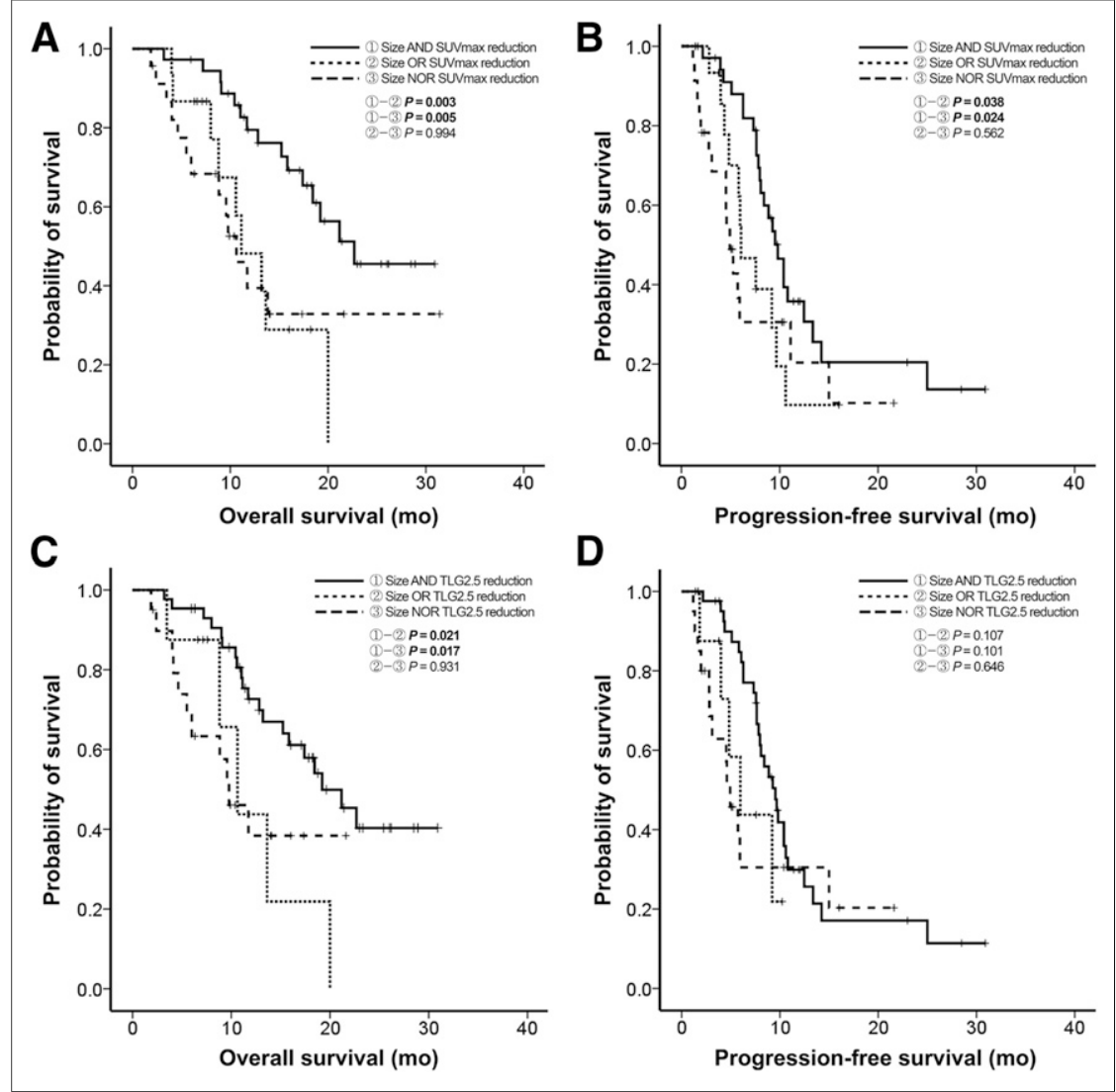

FIGURE 2. Kaplan-Meier curves for OS or PFS based on criteria of $30 \%$ tumor size reduction and $50 \%$ reduction of $\mathrm{SUV}_{\max }(\mathrm{A}$ and $\mathrm{B}$ ) or $60 \%$ reduction of TLG2.5 (C and D). Cutoff values were determined using linear regression equations.

both $30 \%$ size and $50 \% \mathrm{SUV}_{\max }$ reduction were the significant factors for better OS and PFS (hazard ratio for OS $=4.41, P<$ 0.001 ; hazard ratio for PFS $=2.58, P=0.002$ ). Both $30 \%$ size and $60 \%$ TLG2.5 reduction were also the significant factors in multivariate analyses (hazard ratio for $\mathrm{OS}=3.87, P=0.001$; hazard ratio for PFS $=2.19, P=0.015)$ (Supplemental Tables 2 and 3 ).

\section{DISCUSSION}

This study was conducted to identify the relationship between changes in tumor metabolism measured by PET and changes in tumor size measured by CT, and to assess its impacts on survival in patients undergoing chemotherapy for AGC. Instead of measuring only the target lesions defined according to the RECIST, all reproducibly measurable lesions larger than $1 \mathrm{~cm}$ were included in this study for exact analyses using a large number of lesions. We also applied the analysis according to the RECIST and found similar results. Generally, the primary gastric lesion is not a suitable candidate for measuring the exact tumor size repeatedly because of its irregular conformation. For that reason, most primary lesions were not included in our analyses.

There was a significant correlation between baseline tumor size and $\mathrm{SUV}_{\max }$ or TLG2.5 in each of the lesions at the pretreatment evaluation. TLG2.5 is calculated by multiplying the $\mathrm{SUV}_{\text {mean }}$ and metabolic tumor volume, and the metabolic tumor volume is, conceptually, similar with tumor size. Therefore, the strong correlation between tumor size and TLG2.5 is a somewhat natural result. However, the correlation of $\mathrm{SUV}_{\max }$ with size in each lesion is an interesting result, and it implies that tumor metabolism increases as each tumor lesion increases in size. The exception was poorly cohesive carcinoma. A few other studies have been conducted in the same context of this result. A study on gastric cancer demonstrated the relationship between $\mathrm{T}$ stage and $\mathrm{SUV}_{\max }$ (15), and another study showed the correlation between the maximal diameter of primary lesion and $\mathrm{SUV}_{\max }$. In the latter study, messenger RNA level of hypoxia-inducible factor $1 \alpha$ was also correlated with $\mathrm{SUV}_{\max }$; therefore, they suggested the mechanism that growth-related hypoxia induces anaerobic glycolysis and increases glucose metabolism (16).

A previous study, which analyzed primary lesions of AGC, found that the $\mathrm{SUV}_{\max }$ of HER2-positive tumors was higher than that of HER2-negative tumors (17). In our study, although there was no significant difference in baseline $\mathrm{SUV}_{\text {max }}$ between HER2-positive and HER2-negative tumors in the analysis including whole lesions, HER2-positive lesions showed higher baseline $\mathrm{SUV}_{\max }$ than did HER2-negative lesions in the analysis including lesions larger than $20 \mathrm{~mm}$. This is because of the tendency of $\mathrm{SUV}_{\max }$ to rise more steeply according to tumor size in HER2-positive tumors than in HER2negative tumors (regression coefficient of $\mathrm{SUV}_{\max }$ change per $1 \mathrm{~mm}$ size change: 0.22 in HER2-positive tumors, 0.08 in HER2-negative tumors, $P<$ 0.001). Poorly cohesive carcinomas showed lower baseline $\mathrm{SUV}_{\max }$ than did adenocarcinomas irrespective of tumor size in our study. Several previous studies have shown results corresponding well with our results, and all of these studies analyzed primary gastric lesions $(9,18$ 20 ). Our findings provide evidence for the difference in $\mathrm{SUV}_{\max }$ between two histologic types in all evaluable lesions.

Previous studies have reported that early metabolic response is related to better prognosis in gastric cancer (21-24). Ott et al. showed that early metabolic response of neoadjuvant chemotherapy within $2 \mathrm{wk}$ is linked to a histopathologic response and favorable prognosis in locally advanced gastric cancer (22). Di Fabio et al. also demonstrated that $\mathrm{SUV}_{\text {max }}$ reduction within $6 \mathrm{wk}$ after chemotherapy was associated with better survival in patients with metastatic gastric cancer (23). Lorenzen et al. showed the correlation between early metabolic response and subsequent size response after palliative chemotherapy in a small number of gastric and esophageal cancer patients (24). However, there was no previous study that evaluated the additive impact of PET and CT on survival with accurate comparison of them at the same time (25).

In this study, SUV $_{\max }$ change to chemotherapy has a definite linear correlation with size change. According to our data, clinicians can expect an approximately $50 \% \mathrm{SUV}_{\max }$ reduction when the lesions showed a $30 \%$ size reduction. This result would be helpful for clinicians when making a decision based on discrepant results of PET and CT after chemotherapy. Better OS and PFS were observed in the patients with a reduction in both size and $\mathrm{SUV}_{\max }$ than in 
patients with a reduction in either size or $\mathrm{SUV}_{\max }$. Although TLG2.5 also showed results similar to $\mathrm{SUV}_{\max }, \mathrm{SUV}_{\max }$ seems to be the more useful parameter because of its stronger linearity with size change and lesser variation. These data suggest that response assessment via a combination of CT and ${ }^{18} \mathrm{~F}$-FDG PET may provide enhanced prediction of prognosis after chemotherapy in AGC.

A limitation of our study is the small sample size. However, this limitation was partly compensated for through the analyses including all evaluable lesions of metastatic gastric cancer. Additional studies with larger sample sizes are needed to validate these results and enable their application in clinical practice.

\section{CONCLUSION}

The reduction in tumor size after chemotherapy has a linear correlation with the reduction in tumor metabolism, and a $30 \%$ size reduction corresponds well with a $50 \% \mathrm{SUV}_{\max }$ reduction in gastric cancer. Considering both changes together could help predict a more accurate prognosis for patients undergoing chemotherapy for AGC.

\section{DISCLOSURE}

This study was supported by Seoul National University Hospital Research Fund (grant 25-2014-0140) to Dr. Do-Youn Oh and by a grant from the Korea Health Technology R\&D Project through the Korea Health Industry Development Institute (KHIDI), funded by the Ministry of Health \& Welfare, Republic of Korea (grant HI14C1072) to Gi Jeong Cheon. No other potential conflict of interest relevant to this article was reported.

\section{ACKNOWLEDGMENTS}

This study was partly presented at the Gastrointestinal Cancers Symposium 2016 held in San Francisco, California, USA.

\section{REFERENCES}

1. Sasaki R, Komaki R, Macapinlac H, et al. $\left[{ }^{18} \mathrm{~F}\right]$ fluorodeoxyglucose uptake by positron emission tomography predicts outcome of non-small-cell lung cancer. $J$ Clin Oncol. 2005;23:1136-1143.

2. Downey RJ, Akhurst T, Ilson D, et al. Whole body 18FDG-PET and the response of esophageal cancer to induction therapy: results of a prospective trial. J Clin Oncol. 2003;21:428-432.

3. Barrington SF, Mikhaeel NG, Kostakoglu L, et al. Role of imaging in the staging and response assessment of lymphoma: consensus of the International Conference on Malignant Lymphomas Imaging Working Group. J Clin Oncol. 2014;32:3048-3058.

4. Wagner AD, Grothe W, Haerting J, Kleber G, Grothey A, Fleig WE. Chemotherapy in advanced gastric cancer: a systematic review and meta-analysis based on aggregate data. J Clin Oncol. 2006;24:2903-2909.

5. Bang YJ, Van Cutsem E, Feyereislova A, et al. Trastuzumab in combination with chemotherapy versus chemotherapy alone for treatment of HER2-positive advanced gastric or gastro-oesophageal junction cancer (ToGA): a phase 3, openlabel, randomised controlled trial. Lancet. 2010;376:687-697.
6. Torre LA, Bray F, Siegel RL, Ferlay J, Lortet-Tieulent J, Jemal A. Global cancer statistics, 2012. CA Cancer J Clin. 2015;65:87-108.

7. Eisenhauer EA, Therasse P, Bogaerts $J$, et al. New response evaluation criteria in solid tumours: revised RECIST guideline (version 1.1). Eur J Cancer. 2009;45: $228-247$.

8. Weber WA, Ott K. Imaging of esophageal and gastric cancer. Semin Oncol. 2004; 31:530-541.

9. Smyth E, Schoder H, Strong VE, et al. A prospective evaluation of the utility of 2-deoxy-2-[ $\left.{ }^{18} \mathrm{~F}\right]$ fluoro-D-glucose positron emission tomography and computed tomography in staging locally advanced gastric cancer. Cancer. 2012;118:5481-5488.

10. Chen J, Cheong JH, Yun MJ, et al. Improvement in preoperative staging of gastric adenocarcinoma with positron emission tomography. Cancer. 2005;103:2383-2390.

11. Ott K, Weber WA, Lordick F, et al. Metabolic imaging predicts response, survival, and recurrence in adenocarcinomas of the esophagogastric junction. J Clin Oncol. 2006;24:4692-4698.

12. Lordick F, Ott K, Krause BJ, et al. PET to assess early metabolic response and to guide treatment of adenocarcinoma of the oesophagogastric junction: the MUNICON phase II trial. Lancet Oncol. 2007;8:797-805.

13. Lorenzen S, von Gall C, Stange A, et al. Sequential FDG-PET and induction chemotherapy in locally advanced adenocarcinoma of the Oesophago-gastric junction (AEG): the Heidelberg Imaging program in Cancer of the oesophagogastric junction during Neoadjuvant treatment: HICON trial. BMC Cancer. 2011;11:266.

14. Baiocchi GL, Marrelli D, Verlato G, et al. Follow-up after gastrectomy for cancer: an appraisal of the Italian research group for gastric cancer. Ann Surg Oncol. 2014;21:2005-2011.

15. Namikawa T, Okabayshi T, Nogami M, Ogawa Y, Kobayashi M, Hanazaki K. Assessment of ${ }^{18} \mathrm{~F}$-fluorodeoxyglucose positron emission tomography combined with computed tomography in the preoperative management of patients with gastric cancer. Int J Clin Oncol. 2014;19:649-655.

16. Takebayashi R, Izuishi K, Yamamoto Y, et al. $\left[{ }^{18} \mathrm{~F}\right]$ Fluorodeoxyglucose accumulation as a biological marker of hypoxic status but not glucose transport ability in gastric cancer. J Exp Clin Cancer Res. 2013;32:34.

17. Ock CY, Kim TY, Lee KH, et al. Metabolic landscape of advanced gastric cancer according to HER2 and its prognostic implications. Gastric Cancer. 2016;19:421430.

18. Mukai K, Ishida Y, Okajima K, Isozaki H, Morimoto T, Nishiyama S. Usefulness of preoperative FDG-PET for detection of gastric cancer. Gastric Cancer. 2006;9:192-196.

19. Yamada A, Oguchi K, Fukushima M, Imai Y, Kadoya M. Evaluation of 2-deoxy$2-\left[{ }^{18} \mathrm{~F}\right]$ fluoro-D-glucose positron emission tomography in gastric carcinoma: relation to histological subtypes, depth of tumor invasion, and glucose transporter-1 expression. Ann Nucl Med. 2006;20:597-604.

20. Stahl A, Ott K, Weber WA, et al. FDG PET imaging of locally advanced gastric carcinomas: correlation with endoscopic and histopathological findings. Eur $J$ Nucl Med Mol Imaging. 2003;30:288-295.

21. Couper GW, McAteer D, Wallis F, et al. Detection of response to chemotherapy using positron emission tomography in patients with oesophageal and gastric cancer. Br J Surg. 1998;85:1403-1406.

22. Ott K, Herrmann K, Lordick F, et al. Early metabolic response evaluation by fluorine-18 fluorodeoxyglucose positron emission tomography allows in vivo testing of chemosensitivity in gastric cancer: long-term results of a prospective study. Clin Cancer Res. 2008;14:2012-2018.

23. Di Fabio F, Pinto C, Rojas Llimpe FL, et al. The predictive value of ${ }^{18}$ F-FDGPET early evaluation in patients with metastatic gastric adenocarcinoma treated with chemotherapy plus cetuximab. Gastric Cancer. 2007;10:221-227.

24. Lorenzen S, Herrmann K, Weber WA, et al. Visualisation of metastatic oesophageal and gastric cancer and prediction of clinical response to palliative chemotherapy using 18FDG PET. Nuklearmedizin. 2007;46:263-270.

25. Rahim MK, Kim SE, So H, et al. Recent trends in PET image interpretations using volumetric and texture-based quantification methods in nuclear oncology. Nucl Med Mol Imaging. 2014;48:1-15. 